Journal of Computer Science 7 (10): 1541-1544, 2011

ISSN 1549-3636

(C) 2011 Science Publications

\title{
Wavelet Analysis and Orthogonal Moments based Classification of Microcalcification in Digital Mammograms
}

\author{
${ }^{1}$ N.V.S. Sree Rathna Lakshmi and ${ }^{2}$ C. Manoharan \\ ${ }^{1}$ Anna University, Coimbatore, \\ ${ }^{2}$ VSA Group of Educational Institutions, Salem, India
}

\begin{abstract}
Problem statement: An important early sign of breast cancer is the clusters of micro calcifications in digital mammograms. To assist radiologists in the diagnosis of mammographic clusters a novel hybrid algorithm has been developed. Approach: A method, for detecting micro calcification in mammograms based on combined feature set with Ant Colony Optimization (ACO) was proposed. The diagonal matrix ' $S$ ' obtained from the Singular Value Decomposition (SVD) of LL band of wavelet transform was used as one of the feature set for classification of mammogram. A new approach for detecting micro calcifications in mammograms employing Jacobi Moments was proposed. The set of Jacobi polynomials were orthogonal and this ensured minimal information redundancy between the moments. Results and Conclusion: Jacobi moments include the properties of well-known Zernike, Legendre and Tchebichef moments. Ant Colony Optimization (ACO) was used for reducing the Jacobi feature set dimensionality through selecting a subset of features that performed well in the classification phase. The selected Jacobi feature set were combined with ' $S$ ' matrix to achieve the better classification rate of over $90 \%$.
\end{abstract}

Key words: Jacobi polynomials, Ant Colony Optimization (ACO), Singular Value Decomposition (SVD), pixel groupings

\section{INTRODUCTION}

Mammography is the only efficient and feasible technique to detect breast cancer especially in the case of minimal tumors. About $30-50 \%$ of breast cancers exhibit deposits of calcium called micro calcifications. Intensive research work has been undertaken in the development of automated image analysis methods to assist radiologists in the identification of abnormalities. The inventory as linear optimal control problem and by the Pontryagin maximum principle is developed Baten and Kamil, (2009), the optimal control problem was solved analytically to obtain the optimal solution of the problem. ACO algorithm for load balancing in distributed systems described Ali et al. (2010). It is fully distributed in which information is dynamically updated at each ant movement.

The Ant Colony Optimization (ACO) for solving solid waste collection problem as a VRPSD model is proposed Ismail and Loh (2009). A set of data modified from the well known 50 customers problems were used to find the route such that the expected traveling cost was minimized. A systematic model of delivering Ready Mixed Concrete (RMC) that optimizes the schedule of dispatching RMC trucks, presented Srichandum and Rujirayanyong (2010).
Many studies have been made on the problem of breast cancer diagnosing based on digital mammograms. The Least Square with Regularization (LSR) method to reconstruct the image and the electrodynamics sensor was used to capture the data that installed around the pipe is described Sayeed et al. (2009).

The implementation of data glove from motion animation towards signature verification problem is described Sayeed et al. (2009), making use of the offered multiple degrees of freedom for each finger and for the hand as well. An an SVD-based technique to extract the feature values of different sensors' locating on corresponding fingers in the signing process and evaluated the results for writer authentication. Ant Colony Optimization (ACO) algorithm for the general combinatorial optimization problem is presented $\mathrm{Yu}$ and Zhang (2009). The model proved that it can converge to one of the optima if only this optimum is allowed to update the pheromone model and that it cannot converge to any of the optima if two or more optima are allowed.

A hybrid approach with Ant Colony Optimization (ACO) and Code Filtering System (CFS) for DSCDMA multiuser detection is proposed $\mathrm{Wu}$ et al. 
(2010). In CFS, the method of Lagrange multipliers is applied to set the threshold in order to make the judgment of the codes of users. A robust quantization based digital image watermarking for copy right protection in DCT-SVD domain is described Abdulfetah et al. (2009). The watermarking algorithm which combines both merits of the algorithm based on Discrete Cosine Transform (DCT) and algorithm based on Singular Value Decomposition (SVD). An efficient method for electrical charge tomography image reconstruction has been introduced by Brahmat et al. (2010).

\section{MATERIALS AND METHODS}

The proposed system: The block diagram of classification of microcalcification in mammograms based on combined feature set with SVM is shown in Fig. 1.

Feature extraction: Feature extraction involves simplifying the amount of resources required to describe a large set of data accurately. In the proposed method two features are combined for the classification of micro calcification in the mammogram. They are ' $\mathrm{S}$ ' feature and the Jacobi moments.
'S' feature extraction: Two-dimensional discrete Haar wavelet transform is applied to the given ROI image. It decomposes an input image into four sub-bands, one average component (LL) and three detail components (LH, HL, HH). Then SVD is applied to the LL sub band only. After applying SVD to the LL band of Wavelet Transform, three rectangular matrices $\mathrm{S}, \mathrm{U}$ and $\mathrm{V}$ are obtained. $\mathrm{S}$ is a diagonal matrix which contains the square root Eigen values from $\mathrm{U}$ or $\mathrm{V}$ in descending order is selected and stored it separately in the feature set. The original ROI image is shown in Fig. 2a.

The Wavelet Decomposition image is shown in Fig. 2 (b) and the LL band is shown in Fig. 2(c).

Jacobi moments extraction: The Jacobi moments are calculated for the given ROI image. Since the image has $256 \times 256$ sizes, it produces high number of Jacobi moments. Ant Colony Optimization is used for reducing the Jacobi feature set by selecting a subset of features which contains 10 moments that performs very well in the classification phase. And all the 10 moments are stored combined with the ' $S$ ' feature. This combined set is used in the classification phase.

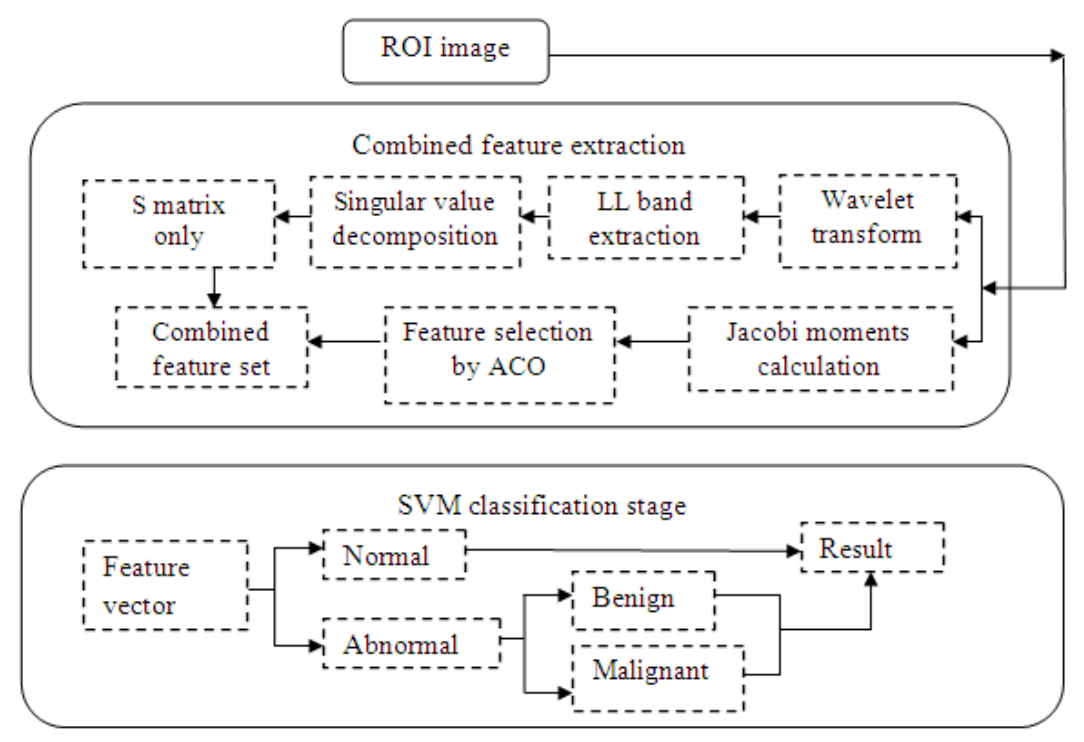

Fig. 1: Block diagram of the proposed method based on combined feature set with SVM

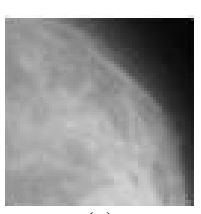

(a)

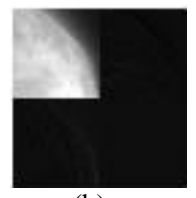

(b)

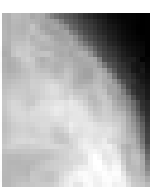

(c)

Fig. 2: (a) Input image (b) Wavelet Transform Image (c) LL Band image 1542 


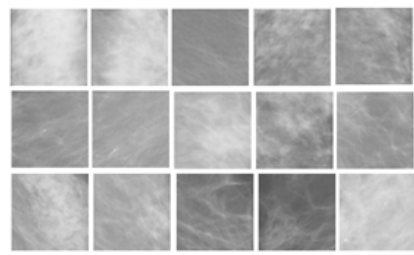

(a)

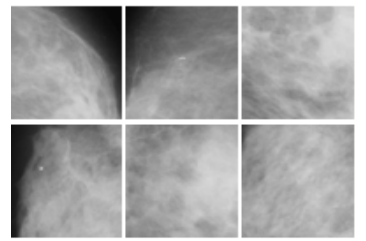

(b)

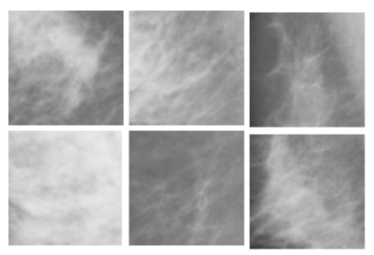

(c)

Fig. 3: (a) Training set-normal image, (b) training setbenign image, (c) training Set-Malignant Image

Table 1: Number of Training and Testing samples for $1^{\text {st }}$ stage classifier

\begin{tabular}{lll}
\hline Type of image & No of training images & No of testing images \\
\hline Normal & 30 & 194 \\
Abnormal & 12 & 25 \\
\hline
\end{tabular}

Table 2: Number of Training and Testing samples for $2^{\text {nd }}$ stage classifier

\begin{tabular}{lll}
\hline Type of image & No of training set & No of Testing set \\
\hline Benign & 6 & 12 \\
Malignant & 6 & 13 \\
\hline
\end{tabular}

Classification phase: In the proposed method SVM is used as a classifier. Classification phase executes two phases. In the first one, the classifier is applied to classify mammograms into normal and abnormal cases. Then the mammogram is considered abnormal if it contains tumor (microcalcification). Finally, the abnormal mammogram is classified into malignant or benign in the second stage. In this classification stage, SVM classifier in every phase is trained at specific number of training set in each category.

1st stage classifier: In the first stage, classifier is tested for normal or abnormal images based on combined feature set of "S" matrix and the Jacobi moments features. Then the calculated combined feature set of training images is first trained with the SVM classifier and then tested for all the images including training images for the classification.

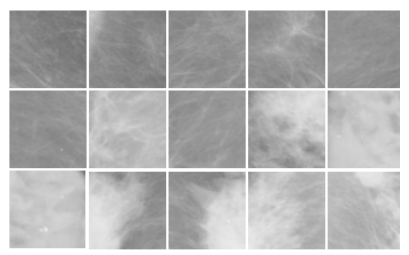

(a)

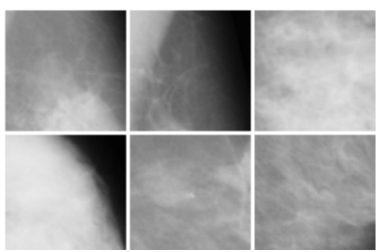

(b)

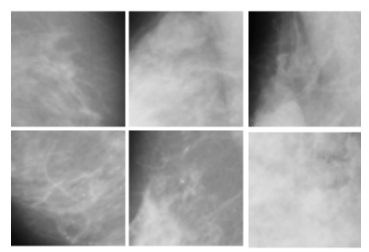

(c)

Fig. 4: (a) Testing set- normal image, (b) testing setbenign image, $c$ ) testing set-malignant image

The number of training and testing images for the 1st stage is given in Table 1. The Training set for Normal Image, Benign Image and Malignant Image is shown in Fig. 3a-c respectively.

2nd stage classifier: In this stage, the abnormal images from the 1st stage classifier are further classified into Benign or Malignant. The calculated combined feature set of training images is first trained with the SVM classifier and then tested for all the images including training images for the classification of Benign/Malignant. The number of training and testing images for the 2 nd stage is given in Table 2. The Testing set for Normal Image, Benign Image and Malignant Image is shown in Fig. 4a-c respectively.

\section{RESULTS AND DISCUSSION}

The experiments are conducted on the MIAS database (MIAS database, 0000). In MIA's database, there are 194 normal images and 25 micro calcification images available. All the images are considered for the classification test. The classification rate obtained using the combination of SVD feature sets and Jacobi feature sets results are show in Table 3. The GUI Implementation of the proposed system is shown in Fig. 5. 


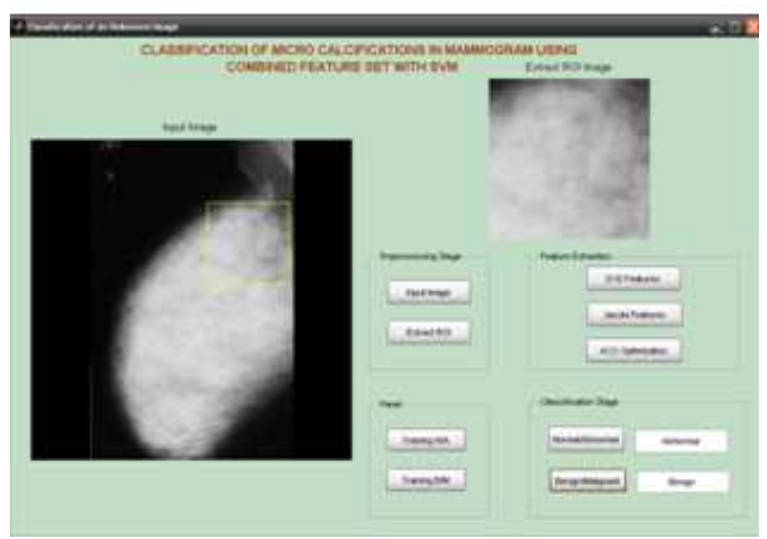

Fig. 5: GUI Implementation of the proposed system

\begin{tabular}{lc} 
Table 3: Classification Result of the proposed system \\
\hline Image type & $(\%)$ classification rate \\
\hline Normal & 92.78 \\
Abnormal & 92.00 \\
Benign & 91.67 \\
Malignant & 92.31 \\
\hline
\end{tabular}

\section{CONCLUSION}

This study presents a novel method for the classification of mammogram microcalcifications using combined feature set with ant colony optimization to improve classification accuracy with a small and appropriate Jacobi feature subset. Our method is capable of classifying the given mammogram into Normal/Abnormal as well as Benign/Malignant in case of Abnormal. Our method is successful in detecting the micro calcification in the mammogram.

The experimental results indicate that the hybridized approach can correctly select the discriminating input features of Jacobi moments and also achieve high classification accuracy. Furthermore, a clinically useful system for breast cancer detection must be able to detect the breast cancers, not just micro calcifications. Our future work is to extend the combined feature set for the detection of mass classification.

\section{REFERENCES}

Baten, M.A. and A.A. Kamil, 2009. An optimal control approach to inventory-production systems with weibull distributed deterioration. J. Math. Stat., 5: 206-214. DOI: 10.3844/JMSSP.2009.206.214

Ali, A.D., M.A. Belal and M.B. Al-Zoubi, 2010. Load balancing of distributed systems based on multiple ant colonies optimization. Am. J. Applied Sci., 7: 428-433. DOI: 10.3844/AJASSP.2010.428.433

Ismail, Z. and S.L. Loh, 2009. Ant colony optimization for solving solid waste collection scheduling problems. J. Math. Stat., 5: 199-205. DOI: 10.3844/JMSSP.2009.199.205

Srichandum, S. and T. Rujirayanyong, 2010. Production scheduling for dispatching ready mixed concrete trucks using bee colony optimization. Am. J. Eng. Applied Sci., 3: 7-14. DOI: 10.3844/AJEASSP.2010.7.14

Brahmat, M. F., M.D. Isa, K. Jusoff, T.A. Hussin and S. M. Rozali, 2010. Image reconstruction algorithm for electrical charge tomography system. Am. J. Applied Sci., 7: 1254-1263. DOI: 10.3844/AJASSP.2010.1254.1263

Shohel Sayeed, Nidal S. Kamel and Rosli Besa, 2009. A Novel Approach to Dynamic Signature Verification Using Sensor-Based Data glove. American Journal of Applied Sciences, 6: 233-240. DOI: 10.3844/AJASSP.2009.233.240

$\mathrm{Yu}, \mathrm{X}$. and T. Zhang, 2009. Convergence and runtime of an ant colony optimization model. Inform. Technol. $\quad$ J., $\quad$ 8: $354-359$. DOI: 10.3923/ITJ.2009.354.359

$\mathrm{Wu}, \mathrm{Z}$., Y. Kuang, N. Zhao and Y. Zhao, 2010. A hybrid CDMA multiuser detector with ant colony optimization and code filtering system. Inform. Technol. $\quad$ J., $\quad 9$ : $818-824$. DOI: $10.3923 /$ ITJ.2010.818.824

Abdulfetah, A., X. Sun and H. Yang, 2009. Quantization based robust image watermarking in DCT-SVD domain. Res. J. Inform. Technol., 1: 107-114. DOI: 10.3923/RJIT.2009.107.114 